\title{
Influence of Temperature on the Measuring Accuracy of Devices Based on Surface Plasmon Resonance Phenomenon
}

\author{
Hanna V. Dorozinska1, Tatyana A. Turu1, Olga M. Markina1, Glib V. Dorozinsky², \\ Volodymyr P. Maslov² \\ ${ }^{1}$ National Technical University of Ukraine "Igor Sikorsky Kyiv Polytechnic Institute", Kyiv, Ukraine \\ ${ }^{2} \mathrm{~V}$. Lashkaryov Institute of Semiconductor Physics NAS of Ukraine, Kyiv, Ukraine \\ Email:vpmaslov@ukr.net
}

How to cite this paper: Dorozinska, H.V., Turu, T.A., Markina, O.M., Dorozinsky, G.V. and Maslov, V.P. (2018) Influence of Temperature on the Measuring Accuracy of Devices Based on Surface Plasmon Resonance Phenomenon. Modern Instrumentation, 7, 1-10.

https://doi.org/10.4236/mi.2018.71001

Received: December 21, 2017

Accepted: January 23, 2018

Published: January 26, 2018

Copyright $\odot 2018$ by authors and Scientific Research Publishing Inc. This work is licensed under the Creative Commons Attribution International License (CC BY 4.0).

http://creativecommons.org/licenses/by/4.0/

\begin{abstract}
The main reasons for the occurrence of temperature error and perspective directions of decreasing of its value are presented by improving the device design based on the phenomenon of surface plasmon resonance (SPR) and numerical methods of processing the results of measurements by this device. The most essential influence on changes in results of measurements can be rendered by temperature changes in the analyte refractive index. It is shown that the application of the integrated temperature stabilization of the device with the test substance, and numerical processing of the measurement results for compensation of temperature changes of the test substance and the use of film heaters it is possible to reduce the measurement error of the refractive index of the analyte at least 30 times from $2.4 \times 10^{-4}$ to $7.6 \times 10^{-6}$.
\end{abstract}

\section{Keywords}

Surface Plasmon Resonance, Temperature Factor, Measuring Accuracy

\section{Introduction}

One of the promising directions in developing analytical devices is application of surface electromagnetic waves from the optical range in 1D - 3D nanostructures as well as observation of surface plasmon resonance phenomena arising in this case. Optical measurements based on the phenomenon of surface plasmon resonance (SPR) are widely used in chemical and biological analyses that are found on registration of molecular adsorption in different media from gases to liquids [1] [2] [3] [4] [5] and the solids (e.g. inorganic solid particles [6] and the organic 
film Langmuir-Blodgett [7]). Diagnostic devices that operate using the SPR phenomenon possess high sensitivity to low concentrations of studied substances, which enables to use them as precise analytical tools in laboratory investigations performed in food, chemical and pharmaceutical industry, agriculture, medicine and ecology [8] [9] [10] [11]. At the same time to obtain relevant results of the study should be provided high measurement accuracy of the SPR-instrument. The measurement accuracy of the SPR devices is ensured by the evaluation of the factors influencing the magnitude of error of the measurement result such devices. The wavelength of excitation of the surface plasmons, the topology of the sensitive element of the device and the temperature change elements of the optical scheme of the instrument and analyte are the main factors. The impact of temperature is one of important factors of influence. It determines the half of the size error of the measurement result. Therefore, the analysis of mechanisms of temperature influence on the measurement devices on the basis of SPR and the development of methods and means of reducing this influence is an urgent task.

Here the authors present the basic causes of temperature error. It is shown a promising direction of reducing its size by improving the design of SPR-device and numerical methods for processing results of measurements by this device.

\section{Surface Plasmon Resonance Devices}

The most significant advantage of SPR techniques is their capability of label-free real-time monitoring of biomolecular interactions that opened up the way to develop a new concept for analysis of functional properties of biomolecules, the affinity biosensor technology [12]. The SPR based technique can be applied to the measurement of the ligand-receptor and enzyme-substrate interactions, for label-free immunoassays, in polyclonal antibody characterization, epitope mapping, protein conformation studies, DNA hybridization etc. Apart from biomedical researches, it can also be used in the field of material science for studying interfacial physical and chemical processes: electrochemical reactions, corrosion, adsorption, heterogeneous catalysis and many others. The SPR biosensor advantage as compared with more conventional methods, for instance the enzyme linked immunoassay (ELISA), consists in the real-time monitoring of intermolecular interactions and performing fast analysis without need of enduring sample incubation.

Great advantage of SPR-refractometers "Plasmon" series produced by V. Lashkaryov Institute of Semiconductor Physics NAS of Ukraine are block-modular design, which makes it easy to update the device for various applications and possibility investigation gaseous media [13]. This devices provides measurements of the total SPR curve in the angle configuration that corresponds to the Kretchmann optical geometry with mechanical scanning the angle of incidence within the range 17 degrees in air (12 degrees in glass) and accuracy 5 arc seconds as well as the possibility to make absolute calibration by the angle. Excitation of 
surface plasmons in thin gold film is realized using the p-polarized laser beam and a glass coupling prism. It allows registering SPR curves within the range of incidence angles 56 to 74 degrees (in glass). The wide range of angles for scanning allows performing quantitative measurements of a full reflection curve including the total internal refraction and SPR angles for liquids with various refraction indexes-from 1.33 (water) up to 1.50 (UV-glue). Refractometers Plasmon series allows to measure the refractive index over a wide range from $5 \mathrm{RU}$ (relative units) to $130,000 \mathrm{RU}$ with an accuracy $\pm 3 \times 10^{-6} \mathrm{RIU}$ (refractive index unit) [14].

SPR devices "Plasmon" series used two measurement modes: Multiple and Slope. In Multiple mode, periodically measuring the angular position of the minimum of a characteristic reflection $R(\theta)$, and Slope-periodic measurement of the intensity of the reflected light at a constant angle of its fall (Figure 1). The change in the refractive index of the analyte causes a shift in the characteristics of the reflection $R(\theta)$ from the starting position (red curve) to the end position (black curve).

In Mode Multiple is determined by this shift, is cycle of angular scanning and simultaneous measurement of the intensity reflected from the sensing element of light and is measured the amount of angular displacement $\Delta \theta \mathrm{min}$. In the mode of Slope is first measured all the SPR curve, and then the change in the reflected light intensity (reflectance $\Delta R$ ) at constant angle of incidence. The magnitude of changes in $\Delta \theta$ min or $\Delta R$ are calculated change of the refractive index $\Delta \mathrm{Na}$ of analyte.

In the series Plasmon devices for decreasing temperature measurement error is used complex stabilization: the required temperature as the test sample and of the device as a whole.

In the subsequent sections review the methods and means to improve the accuracy of refractometers Plasmon.

\section{Influence of the Temperature Factor on the Measuring Accuracy}

The analysis of literature sources showed that enhancement of measurement

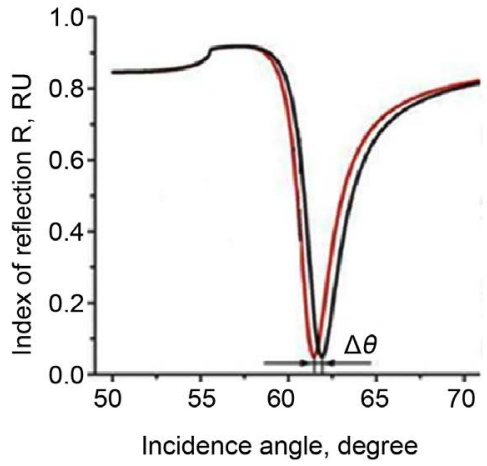

(a)

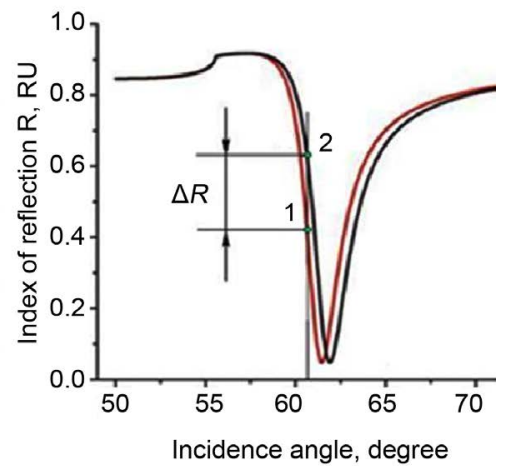

(b)

Figure 1. Ways of measuring the SPR device (a) - the displacement of the $\Delta \theta$ minimum characteristics $R(\theta)$ (Multiple mode), (b) - intensity changes $\Delta R$ (Slope). 
accuracy as well as reliability and efficiency of devices can be provided by temperature stabilization of the measuring cell [15] [16] [17]. The influence of temperature on the accuracy of SPR-devices can be expressed via the absolute error of measurement results caused by temperature changes of refractive index inherent to In the series Plasmon devices for decreasing temperature measurement error is used complex stabilization: the required temperature as the test sample and of the device as a whole.

In the subsequent sections review the methods and means to improve the accuracy of refractometers Plasmon. The temperature of studied liquid or gas medium placed above the sensitive element of the analytical device operating as based on the SPR phenomenon. When carrying out these measurements, one of the problems is to provide an allowable error for the measured value. The error value of measurement results is essentially influenced by temperature oscillations both of the investigated object (liquid or gas) and all the measuring equipment. It is related with temperature changes in ambient medium, heating the measuring equipment, and in some cases with chemical processes in the studied substances, when the heat energy can be evolved or absorbed. During measurements, this error can change as a consequence of the difference in temperature at the beginning of measurements and after their completion. It follows from the mentioned above that it is necessary to determine the reasons for temperature errors in optical measurements based on SPR and investigate experimentally the influence of thermal stabilization that provides reducing these errors when performing measurements in various media.

The value of this error depends on the range of temperature changes in the course of measurements as well as values of refractive index temperature coefficients of optical elements and analyte. The principal elements of optical scheme for exciting the SPR, which have the main effect on the value of this error, are the coupling prism and sensitive metal layer [18]. Besides, the result of analyte refractive index measurements is influenced by the temperature dependence of laser wavelength. The temperature coefficient for the laser wavelength is approximately $(0.12 \cdots 0.15) \mathrm{nm} / \mathrm{K}$ [19]. Temperature changes of refractive index $\mathrm{n}$ and extinction coefficient $\mathrm{k}$ inherent to sensitive element metal layer are, first of all, related with decreasing the density of charge carriers (electrons) in it, which is conditioned by thermal volume expansion of the metal layer and thermal vibrations of the metal crystalline lattice. The density of electrons in metal layer is in inverse proportion to temperature and the coefficient of thermal volume expansion [17]. Temperature changes of the coupling prism refractive index are determined by the value of the temperature coefficient inherent to material from which it is made of. In the devices of Plasmon series, coupling prisms are made of glasses of $K 8\left(n_{P}=1.5145\right)$ and $F 1\left(n_{P}=1.6154\right)$ types for measuring gas-like and liquid analytes, respectively. The temperature coefficients of refraction indexes for these types of glasses are as follows: $1.2 \times 10^{-6} \mathrm{~K}^{-1}$ for type $\mathrm{K} 8$ and $3 \times$ $10^{-6} \mathrm{~K}^{-1}$ for $\mathrm{F} 1$ [20]. For the distilled water $\left(\mathrm{n}_{\mathrm{A}}=1.3314\right)$, as a liquid analyte, the 
temperature coefficient of refractive index is equal $-1 \times 10^{-4} \mathrm{~K}^{-1}$, while that of dried air $\left(\mathrm{n}_{\mathrm{A}}=1.00028\right)$ is close to $-2.5 \times 10^{-5} \mathrm{~K}^{-1}$ [21].

Being based on the known values of temperature coefficients for the elements of SPR-device optical scheme, one can draw a conclusion that the most essential influence on changes in results of measurements can be rendered by temperature changes in the analyte refractive index.

\section{Temperature Influence Decreasing Methods}

All commercial analytical devices based on SPR contain very complex and expensive systems for thermostatic control. This is not always convenient to provide the necessary degree of thermal stability, which depends on the rate of endo- or exothermic chemical reactions in a measuring cell as well as on an ambient temperature. The miniaturization of devices makes them more user-friendly and extends the sphere of their application. However, the miniaturization worsens temperature schedules of the operation of devices because of localization that entails overheating and thereby making the temperature errors of measurement results more appreciable. Tools used in the past such as contact probes and thermocouples could not provide the thermal data quickly or ensure cost-effectiveness. Thanks to the availability of thermal imaging systems, today an engineer can make the requisite analysis timely without delaying product development schedules or interfering in production processes. To improve the device construction, it is insufficient to have information about temperature coefficients for components of its optical scheme, but it is also necessary to know main sources for heat release. With this aim, it was offered and experimentally checked the way to determine these sources by using the thermo vision method. In work [22] authors were detected thermal sources and study the process of spreading the thermal field in the SPR-refractometer Plasmon series, so as to make it more precise and reliable. The main heat source in the electronic unit of refractometer was microcontroller (Figure 2).

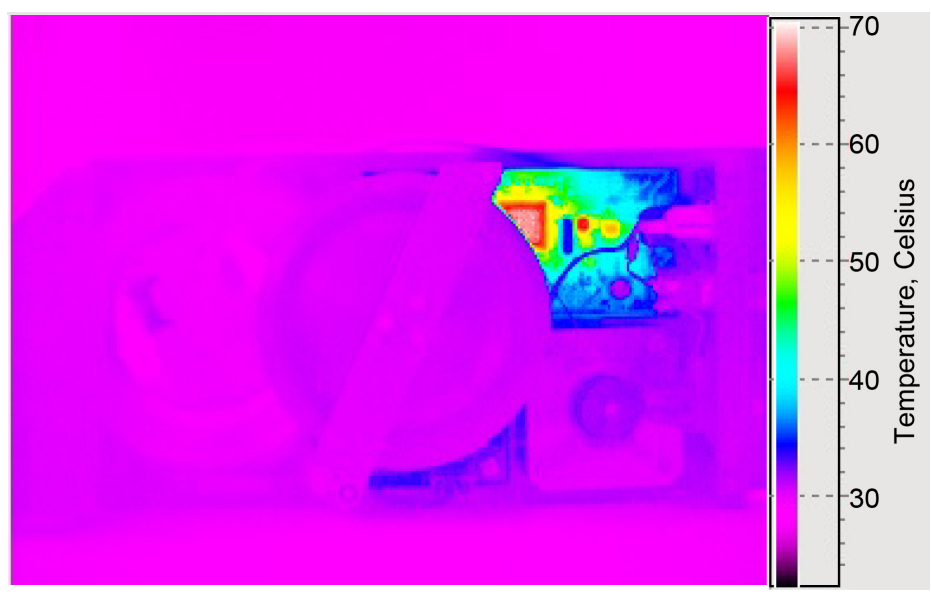

Figure 2. The thermal image of the electronic control unit board with microcontroller. The main heat source is the microcontroller (temperature equal $70^{\circ} \mathrm{C}$ ) [22]. 
Microcontroller temperature has increased from $26^{\circ} \mathrm{C}$ to $70^{\circ} \mathrm{C}$ over the course of $40 \mathrm{~min}$. device operation. Given information make enable to abraded the device Plasmon series to decrease temperature influence by self heating. The device was equipped with air conditioning system for the microcontroller and semiconductor laser as well as to perforate the case for the purpose of facilitating heat removal. Being based on the previous results of investigations by authors was developed and manufactured the thermostat that enabled to keep the set temperature with rather high stability. Was offered thermo stating not only of the studied object but also of all the measuring equipment including tanks with the studies substances [23]. This approach enabled to minimize the temperature error in measurement results and, in addition, temperature loading the measuring equipment, which was prolonged its functioning term. The SPR-refractometer was placed into the thermostat operation chamber where a constant temperature was kept, values of which could be regulated within the range +10 up to $+40^{\circ} \mathrm{C}$ with the step $0.1^{\circ} \mathrm{C}$.

The results of experiments allowed ascertaining that temperature stabilization of measuring equipment reduces the absolute temperature error for the analyte refractive index: in the case of gas-like analytes by 18 times (from $7 \times 10^{-6}$ down to $3.8 \times 10^{-7}$ ), while for the liquid ones-by 3.2 times (from $2.5 \times 10^{-5}$ down to $\left.7.6 \times 10^{-6}\right)$. This decrease of the temperature error due to stabilization of temperature for the device and analyte is explained by reduction of the temperature drift observed for the reflection characteristic minimum when measuring the kinetics. To decrease the error in measurements of refractive index, which is caused by heating the device or by growth of the ambient temperature, it seems purposeful to use compensation of this temperature effect [24]. To compensate the temperature drift of the SPR curve minimum, by authors was used mathematical processing the measurement results. Compensation was provided by taking into account the temperature coefficient of the analyte (distilled water) refraction index that is equal to TKn $\left(\mathrm{H}_{2} \mathrm{O}\right)=-1.15 \times 10^{-4} \mathrm{~K}^{-1}$ as well as temperature changes of water during measurements, which were determined by using the thermo resistor built-in into the case of flow-through measuring cell in the SPR device. Kinetics of the operation point without any compensation of temperature changes observed in the studied substance (1) and with compensation (2) (Figure 3).

The sharp increase in the operation point position at the beginning of the plot (2) is related with inertness of heat transfer processes from the liquid to thermo resistor. The SPR response to temperature changes was faster than that of thermo resistor, which resulted in overcompensation. Elimination of this undesirable phenomenon was provided using the second (reference) channel of device by determination of the difference signal between measuring channels as well as by additional averaging (with account of 10 sequential experimental data). This way allows not only eliminating this over-compensation but additional 12-fold reducing the amplitude of the noise track from $\delta \mathrm{N}_{\mathrm{L}}=3.14 \times 10^{-5}$ down to $\delta \mathrm{N}_{\mathrm{L}}=$ 


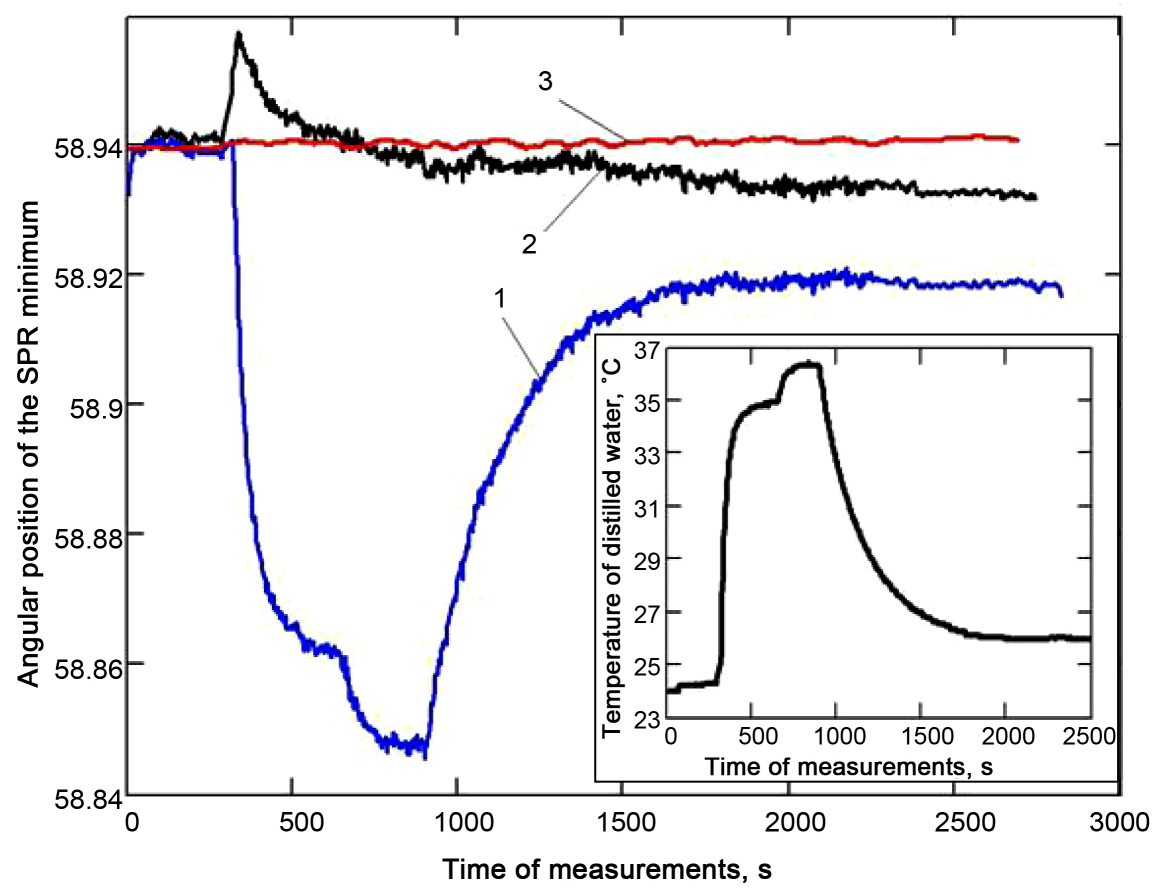

Figure 3. Kinetics of the SPR minimum shift for distilled water when changing its temperature from $24^{\circ} \mathrm{C}$ up to $36.6^{\circ} \mathrm{C}$ without thermal compensation (1), with it (2) and with additional averaging by the data of 10 sequential measurements (3) [24].

$2.7 \times 10^{-6}$. In this case, the measurement error without compensation was $2.4 \times$ $10^{-4}$ and with it was $8.7 \times 10^{-5}$, while with compensation and additional averaging by 10 sequential data of measurements was $2.1 \times 10^{-5}$. Thus thermal compensation decreases the measurement error for the analyte refraction index at least by 3 times, which enables to essentially increase the device accuracy.

To provide wide range of temperature control and fast response of heaters (coolers), one should increase the electric power consumed by them. However, it results in increasing dimensions and consumed power as well as time for transfer heat from a heater (thermo delay). Thermal compensation decreases only the temperature drift, and it is efficient only for stationary temperature regimes. Temperature compensation is effective when we know the nature of the analyte. In the work [25] authors proposed to use ITO (indium tin oxide) film heater. Composite thin films ITO $\left(\mathrm{In}_{2} \mathrm{O}_{3}-20 \%\right.$; $\left.\mathrm{SnO}-80 \%\right)$ were prepared using magnetron sputtering onto glass substrates of the thickness $1 \mathrm{~mm}$ and dimensions $18 \times$ $14 \mathrm{~mm}$. The thickness of deposited layer was determined using atomic force microscopy. At the final stage of formation, this thickness reached the value $50 \mathrm{~nm}$. When studying the thin ITO films as heaters, we determined the consumed power by using the measured current-voltage characteristics with simultaneous measuring the temperature augmentation caused by their self-heating. The voltage on the sample was applied from a power supply $(10 \mathrm{~W})$ with fine adjustment of the output voltage within the range 1.5 to $30 \mathrm{~V}$ and uncertainty $\pm 0.1 \mathrm{~V}$. The same measurements were performed for the glass substrate without ITO film but heating the studied substance by using a constantan wire resistive heater 
(resistance $42 \mathrm{Ohm}$ at the temperature $\mathrm{T}=20^{\circ} \mathrm{C}$ ) built-in into the measuring cell of the SPR-device, the supply voltage of the heater being $12 \mathrm{~V}$. Comparison of the consumed power as well as the error of measurements caused by the temperature drift of the operation point were performed at the given temperature $36.6^{\circ} \mathrm{C} \pm 0.2^{\circ} \mathrm{C}$. The error of measurements was determined as the value of operation point drift, which was expressed using the values of refraction index for the studied air for the interval of measurements equal to $1 \mathrm{~min}$. When the voltage reached its maximum value $30 \mathrm{~V}$ and consumed power- $1.5 \mathrm{~W}$, the film and substrate were heated from $21.5^{\circ} \mathrm{C}$ up to $60.5^{\circ} \mathrm{C}$ for only $5 \mathrm{~min}$. At the same time, to heat the only glass substrate (without ITO film) with the resistive heater up to $55^{\circ} \mathrm{C}$, it requires the power $3.4 \mathrm{~W}$ and voltage $12 \mathrm{~V}$. The thin-film heaters based on ITO consume more than 2-fold lower power in the case of the same heating the studied substance as compared with that for bulk resistive heaters. The temperature drift of the SPR-curve minimum was by 1.8 times lower for film heater than that for the resistive heater, i.e., $3.6 \times 10^{-6} \mathrm{~min}^{-1}$ against $6.5 \times$ $10^{-6} \mathrm{~min}^{-1}$. This fact is related with lower inertness of the processes of heat transfer from thin film as compared with that of the bulk heater heat transfer.

Existing technical solutions for decreasing the effect of the temperature factor can improve the accuracy of measurement at least three orders of magnitude by reducing the error of measurement of the refractive index of analyte from $2.4 \times$ $10^{-4}$ to $7.6 \times 10^{-6}$.

\section{Conclusions}

Analytical instruments based on SPR phenomena are highly sensitive to low concentrations of studied substances, which allow their usage in laboratory research conducted in the food, chemical and pharmaceutical industry, agriculture, medicine and ecology. The effect of temperature is one of important factors of influence and determines the half of the size error of the measurement result. Known technical solutions to reduce the magnitude of the temperature error of the measuring result SPR-devices, namely:

1) Integrated thermal stabilization of the instrument and the containers with the test substance, to reduce an absolute temperature measurement error of the refractive index of analyte: in case of gaseous analytes by 18 times (from $7 \times 10^{-6}$ to $3.8 \times 10^{-7}$ ), for liquid-in 3.2 times (from $2.5 \times 10^{-5}$ to $7.6 \times 10^{-6}$ ).

2) The usage of film heaters in the system to maintain the temperature. For example, film heaters based on ITO consume more than 2 times less power for heating the test substance in comparison with bulk resistive heaters. The temperature drift of baseline for the measurement is 1.8 times less for film heater than for a resistive heater, respectively to $3.6 \times 10^{-6} \mathrm{~min}^{-1}$ and $6.5 \times 10^{-6} \mathrm{~min}^{-1}$, which is due to the smaller inertia of heat transfer processes in thin films compared to bulk heater.

3) Application for compensation of temperature changes using numerical processing of the measurement result. Compensation reduces measurement error of the refractive index of the analyte at least 3 times, which increases the 
measurement accuracy of the SPR-device.

So, existing technical solutions for reducing the effect of the temperature factor can improve the accuracy of measurement at least thirty times.

\section{References}

[1] Liedberg, B., Nylander, C. and Lundstrom, I. (1983) Surface Plasmon Resonance for Gas Detection and Biosensing. Sensors and Actuators B: Chemical, 4, 299-304. https://doi.org/10.1016/0250-6874(83)85036-7

[2] Samoylov, A.V., Mirsky, V.M., Hao, Q., Swart, C., Shirshov, Y.M. and Wolfbeis, O.S. (2005) Nanometer-Thick SPR Sensor for Gaseous HCl. Sensors and Actuators B: Chemical, 106, 369-372. https://doi.org/10.1016/j.snb.2004.08.029

[3] Dorozinsky, G., Lobanov, M. and Maslov, V. (2015) Detection of Methanol Vapor by Surface Plasmon Resonance Method. Eastern-European Journal of Enterprise Technologies, 4, 4-7. https://doi.org/10.15587/1729-4061.2015.47079

[4] Matsubara, K., Kawata, S. and Minami, S. (1988) Optical Chemical Sensor Based on Surface Plasmon Measurement. Applied Optics, 27, 1160-1163.

https://doi.org/10.1364/AO.27.001160

[5] Gridina, N., Dorozinsky, G., Khristosenko, R., Maslov, V., Samoylov, A., Ushenin, Yu. and Shirshov, Yu. (2013) Surface Plasmon Resonance Biosensor. Sensors \& Transducers Journal, 149, 60-68.

[6] Xu, H. and Kall, M. (2003) Modeling the Optical Response of Nanoparticle-Based Surface Plasmon Resonance Sensors. Sensors and Actuators, 87, 244-249. https://doi.org/10.1016/S0925-4005(02)00243-5

[7] Rella, R., Siciliano, P., Quaranta, F., Primo, T., Valli, L., et al. (2000) Gas Sensing Measurements and Analysis of the Optical Properties of Poly [3-(butylthio)thiophene] Langmuir-Blodgett Films. Sensors and Actuators B: Chemical, 68, 203-209. https://doi.org/10.1016/S0925-4005(00)00430-5

[8] Dorozinsky, G., Liptuga, A., Gordienko, V., Maslov, V. and Pidgornyi, V. (2015) Diagnostics of Motor Oil Quality by Using the Device Based on Surface Plasmon Resonance Phenomenon. Scholars Journal of Engineering and Technology, 3, 372374.

[9] Minunni, M. and Mascini, M. (1993) Detection of Pesticide in Drinking Water Using Real-Time Biospecific Interaction Analysis. Analytical Letters, 26, 1441-1460. https://doi.org/10.1080/00032719308017424

[10] Dharmalingam, G., Joy, N.A., Grisafe, B. and Carpenter, M.A. (2012) Plasmonics-Based Detection of $\mathrm{H} 2$ and CO: Discrimination between Reducing Gases Facilitated by Material Control. Beilstein Journal of Nanotechnology, 3, 712-721. https://doi.org/10.3762/bjnano.3.81

[11] Yanase, Y., Hiragun, T., Ishii, K., Kawaguchi, T., et al. (2014) Surface Plasmon Resonance for Cell-Based Clinical Diagnosis. Sensors, 14, 4948-4959.

https://doi.org/10.3390/s140304948

[12] Homola, J. (2006) Surface Plasmon Resonance Based Sensors. In: Wolfbeis, O.S., Ed., Springer Series on Chemical Sensors and Biosensors, Springer-Verlag, Berlin, 45-67. https://doi.org/10.1007/5346_014

[13] Dorozinsky, G.V., Maslov, V.P. and Ushenin, Yu.V. (2016) Sensor Devices Based on Surface Plasmon Resonance. Monograph, Kyiv National Technical University of Ukraine "KPI”, Publishing House "Politeknika”, Kyiv, 264.

[14] Dorozinsky, G., Doroshenko, T. and Maslov, V. (2015) Influence of Technological 
Factors on Sensitivity of Analytical Devices Based on Surface Plasmon Resonance. Journal of Sensor Technology, 5, 54-61. https://doi.org/10.4236/jst.2015.52006

[15] Moreira, C.S., Neff, H., Lima, A. and Thirstrup, G. (2008) Temperature-Dependent Sensitivity of Surface Plasmon Resonance Sensors at the Gold-Water Interface. Sensors and Actuators B Chemical, 134, 854-862. https://doi.org/10.1016/j.snb.2008.06.045

[16] Chiang, H.P., Chen, Ch.-M., Yeh, H.T. and Leung, P.T. (2004) Surface Plasmon Resonance Monitoring of Temperature via Phase Measurement. Optics Communications, 241, 409-418. https://doi.org/10.1016/j.optcom.2004.07.045

[17] Özdemir, S.K. and Turhan-Sayan, G. (2003) Temperature Effects on Surface Plasmon Resonance: Design Considerations for an Optical Temperature Sensor. Journal of Light Wave Technology, 21, 805-815. https://doi.org/10.1109/JLT.2003.809552

[18] Dorozinsky, G.V. (2016) Analysis of the Main Errors of Measurement of the Refractive Index of the Analyte by Device "Plasmon-6". East European Scientific Journal, 5, 54-61.

[19] Bayborodyn, Yu.V. (1988) Fundamentals of Laser Technics. 2nd Edition, Vyshcha Shkola, Kyiv, 383.

[20] Hlaholyev, S.P. (1934) Quartz Glass. Property, Production, Appliance, GHTI, Moscow, 214.

[21] Ravdel, A.A. and Ponomarova, A.M. (1998) Short Guide Physico-Chemical Values. 8th Edition, Special Literature, St. Petersburg, 232.

[22] Dorozinsky, G., Dunaevsky, V. and Maslov, V. (2013) Thermal-Vision Method of Investigations and Control of Device Based on Surface Plasmon Resonance. Universal Journal of Control and Automation, 2, 34-39.

[23] Dorozinsky, G., Maslov, V., Samoylov, A. and Ushenin, Yu. (2013) Reducing Measurement Uncertainty of Instruments Based on the Phenomenon of Surface Plasmon Resonance. American Journal of Optics and Photonics, 1, 17-22. https://doi.org/10.11648/j.ajop.20130103.12

[24] Ushenin, Yu.V., Maslov, V.P., Dorozinsky, G.V., Turu, T.A. and Kachur, N.V. (2016) Application of Temperature Sensors for Improving the Device Based on the Phenomenon of Surface Plasmon. Sensor Electronics and Microsystem Technologies, 13, 33-40. https://doi.org/10.18524/1815-7459.2016.1.70293

[25] Maslov, V.P., Kachur, N.V. and Dorozinsky, G.V. (2016) Investigation of Sensors Based on ITO Nanofilms in SPR-Devices. American Journal of Optics and Photonics, 4, 20-24. https://doi.org/10.11648/j.ajop.20160403.11 